\title{
Preparation and Characterization of Modified Polycarbonate/ABS Blends
}

\author{
Wenfa Dong ${ }^{1,2}$, Ruogu Tang ${ }^{1,2}$, Dingfang Chen $^{3}$
}

\author{
1. Qingdao Changfeng Plastic Co.LTD. Qingdao, China. 266042 \\ 2. School of Polymer Science and Engineering, Qingdao University of Science and \\ Technology. Qingdao, China. 266042 \\ 3. Ningbo Beishi Fishing Petrol Co.LTD. Ningbo, China.315204
}

\begin{abstract}
Polycarbonate based blends have been widely used in plastic engineering and industry, the chemical compositions of the blends directly determined the mechanical properties and performance in real applications. In this study, the polycarbonate (PC) was blended with styrene-based thermoplastics, including acrylonitrile-butadiene-styrene (ABS), acrylonitrile-styrene-acrylate (ASA), or styrenebutadiene-styrene (SBS) with different mass ratios. Mechanical tests indicated that the appropriate blending could result in good compatibility, and specific improved mechanical strengths, though the thermal stability might be worse due to the lose of integrity and homogeneity, this side effect could be largely diminished if the mass ratio between different compositions was well controlled.
\end{abstract}

Key words: Polycarbonate, ABS, ASA, SBS, mechanical properties 


\section{Introduction}

In the past decades, polycarbonate has become a widely used engineering plastic ${ }^{[1]}$. Associated with advantages of mechanical strength, toughness, optical transparency, acid resistance, the polycarbonate has been widely used in many fields ${ }^{[2]}$. For the purpose of improving its overall performances and overcoming the inherent shortages, polycarbonate was often blended with other resins ${ }^{[3-7]}$. Many researches proved that styrene-based thermoplastic is one of the best mate for the blending among various candidates ${ }^{[8-10]}$. It is also well established that the chemical compositions of the polymer blend would directly affect on its compatibility and overall performance in real applications, therefore choosing the appropriate components is the critical step in the blending.

In this work, three type of styrene thermoplastics, acrylonitrile-butadiene-styrene (ABS), acrylonitrile-styrene-acrylate (ASA), and styrene-butadiene-styrene (SBS) were selected to blend with polycarbonate with different mass ratios. The blends were prepared through extrusion and injection molding. The products were characterized of their mechanical and thermal properties for the performance evaluations.

\section{Experiment}

\subsection{Granulation of polycarbonate blends}

The polycarbonate (PC, Qimei Corp, Taiwan) was granulated acrylonitrile-butadiene-styrene (ABS, LG Corp, South Korea), acrylonitrile-styrene-acrylate (ASA, LG Corp), or styrene-butadiene-styrene (SBS, LG Corp) via extrusion molding by using the the twin screw extruder (SHJ-20, provided by Nanjing Giant Machinery Co.,Ltd., China). The mixture formula were shown in Table 1. The temperatures were well controlled and screened as : zone $1: 225^{\circ} \mathrm{C}$, zone $2: 230^{\circ} \mathrm{C}$, zone $3: 235^{\circ} \mathrm{C}$, zone $4: 240^{\circ} \mathrm{C}$, extruder head: $240^{\circ} \mathrm{C}$, polymer melt: $210^{\circ} \mathrm{C}$.

Table 1. Formulation of polycarbonate blend

\begin{tabular}{|c|c|}
\hline Samples & Formula \\
\hline Pure polycarbonate & PC 100g \\
\hline PC/ABS 9010 & PC: $90 \mathrm{~g}, \mathrm{ABS}: 10 \mathrm{~g}$ \\
\hline PC/ABS 8020 & PC: $80 \mathrm{~g}, \mathrm{ABS}: 20 \mathrm{~g}$ \\
\hline PC/ABS 7030 & PC: $70 \mathrm{~g}, \mathrm{ABS}: 30 \mathrm{~g}$ \\
\hline PC/ABS 6040 & PC: $60 \mathrm{~g}, \mathrm{ABS}: 40 \mathrm{~g}$ \\
\hline PC/ASA 9010 & PC: $90 \mathrm{~g}, \mathrm{ASA}: 10 \mathrm{~g}$ \\
\hline PC/ASA 8020 & PC: $80 \mathrm{~g}, \mathrm{ASA}: 20 \mathrm{~g}$ \\
\hline PC/ASA 7030 & PC: 70g, ASA: 30g \\
\hline PC/ASA 6040 & PC: 60g, ASA: 40g \\
\hline PC/SBS 9010 & PC: $90 \mathrm{~g}, \mathrm{SBS}: 10 \mathrm{~g}$ \\
\hline PC/SBS 8020 & PC: $80 \mathrm{~g}$, SBS: $20 \mathrm{~g}$ \\
\hline PC/SBS 7030 & PC: $70 \mathrm{~g}, \mathrm{SBS}: 30 \mathrm{~g}$ \\
\hline PC/SBS 6040 & PC: $60 \mathrm{~g}, \mathrm{SBS}: 40 \mathrm{~g}$ \\
\hline
\end{tabular}

\subsection{Preparation of polycarbonate blends standard test samples}

The PC blends standard test samples were obtained via injection molding (TTI-130F2, Welltec Machinery Ltd, China) ${ }^{[11]}$. The temperatures of different zones were set as a range of: 
injection head: $250-255^{\circ} \mathrm{C}$, zone $1: 230-235^{\circ} \mathrm{C}$, zone $2: 235-240^{\circ} \mathrm{C}$, zone $3: 240-245^{\circ} \mathrm{C}$, zone 4 : $245-250^{\circ} \mathrm{C}$, mold: $85^{\circ} \mathrm{C}$. The injection pressure was set as $10 \mathrm{MPa}$, the holding pressure was set as 11.5MPa and the pressure was kept for 10 seconds.

\subsection{Characterization of polycarbonate blends}

Morphology investigation: a JEOL JSM-6700F scanning electron microscope was used to observe the surfaces of polycarbonate blends.

Mechanical properties tests: all polycarbonate blends were characterized of mechanical properties including tensile strength (GB/T1040.1-2006), Shore D hardness (GB/T2411-2008), flexural strength (GB/T9341-2008), notched impact strength (GB/T1043.1-2008, 7.5J of impact energy).

Thermal properties characterization: the differential scanning calorimetry (DSC, NETZSCH DSC204, Germany) was used to study the thermal properties of polycarbonate blends, the heating and cooling rate was precisely controlled as $20^{\circ} \mathrm{C} / \mathrm{min}$.

Thermal stability characterization: the thermogravimetric analysis were performed on polycarbonate blends to evaluate their thermal stabilities. In addition, the blends were measured of the Vicat (ZWK1302-B, MTS Systems Corporation) softening temperature (GB/T1633-2000, heating rate: $\left.150^{\circ} \mathrm{C} / \mathrm{h}\right)$.

\section{Result and discussion}

\subsection{Morphology}

The Fig.1 showed the SEM images of polycarbonate based samples. The polycarbonate blends, though were not as smooth as pure polycarbonate, displayed an homogeneous and integrated surface morphology without presenting component aggregation and phase separation.

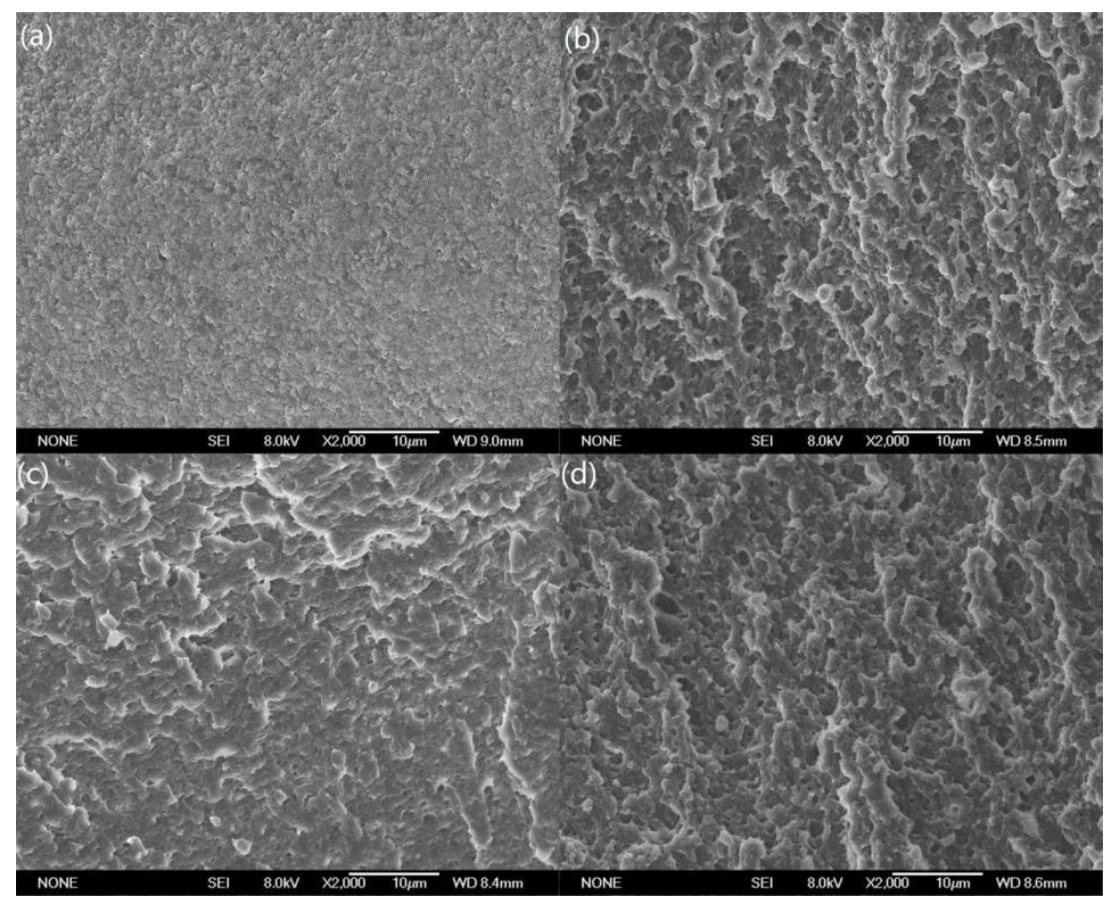

Fig.1. SEM images of (a). Pure polycarbonate; (b). PC/ABS; (c). PC/ASA; (d). PC/SBS. 


\subsection{Mechanical properties}

The mechanical properties of all polycarbonate blends were displayed in Table 2. For these blends, the mechanical properties were critically determined by the types and application amounts of styrene thermoplastics. As shown in the table, PC/ABS blends possessed a good overall mechanical properties, PC/ASA blends possessed the better impact strengths, and PC/SBS blends possessed the better tensile strengths and hardness. The differences of mechanical properties could be attributed the chemical structures of styrene thermoplastics. The ABS has the butadiene components that contains the $\mathrm{C}=\mathrm{C}$ double bonds, when blending with $\mathrm{PC}$, these double bonds could increase the flexibilities of the blends and thus improving the impact strength $^{[12]}$. The ASA has the acrylonitrile components instead of butadiane, the polar nitrile groups on acrylonitrile provide the blends with rigidities so the tensile strengths increased. For the SBS, besides flexible butadiene it also has high content of bulk styrene which result in high tensile strength and hardness. The mass ratios between each components were also significant, overdose of styrene thermoplastics would cause the decrease of mechanical properties of the blends. The reason was that the homogeneity of PC blend would be damaged if the amount of styrene thermoplastics was too high.

Table 2. Mechanical properties of polycarbonate blends

\begin{tabular}{|c|c|c|c|c|}
\hline & $\begin{array}{c}\text { Flexural } \\
\text { strength/MPa }\end{array}$ & $\begin{array}{c}\text { Tensile } \\
\text { strength/MPa }\end{array}$ & $\begin{array}{c}\text { Shore D } \\
\text { hardness }\end{array}$ & $\begin{array}{c}\text { Notched impact } \\
\text { strength/kJ*m }{ }^{-2}\end{array}$ \\
\hline Pure PC & 62.20 & 61.30 & 75 & 26 \\
\hline PC/ABS 9010 & 60.60 & 53.90 & 74 & 38 \\
\hline PC/ABS 8020 & 54.50 & 56.70 & 73 & 45 \\
\hline PC/ABS 7030 & 55.60 & 54.80 & 72 & 40 \\
\hline PC/ABS 6040 & 53.70 & 56.20 & 74 & 29 \\
\hline PC/ASA 9010 & 60.40 & 53.10 & 73 & 32 \\
\hline PC/ASA 8020 & 53.70 & 52.90 & 71 & 33 \\
\hline PC/ASA 7030 & 53.00 & 53.10 & 70 & 32 \\
\hline PC/ASA 6040 & 50.70 & 49.90 & 75 & 29 \\
\hline PC/SBS 9010 & 64.00 & 61.90 & 77 & 34 \\
\hline PC/SBS 8020 & 66.30 & 62.70 & 78 & 38 \\
\hline PC/SBS 7030 & 67.40 & 63.50 & 78 & 36 \\
\hline PC/SBS 6040 & 66.90 & 63.50 & & 73 \\
\hline
\end{tabular}

\subsection{Thermal properties}

The DSC figure was displayed in Fig.2, the blends displayed two endothermic peaks, indicating two glass transition points of the blends, the higher one belonged to the PC components. The two glass transitions indicated that in blends, the two separated phases have been formed. In addition, the glass transition point of polycarbonate was found to be affected by the existence of styrene thermoplastics, that might be caused by the intermolecular forces between two components that affect on the polycarbonate segments movements. 


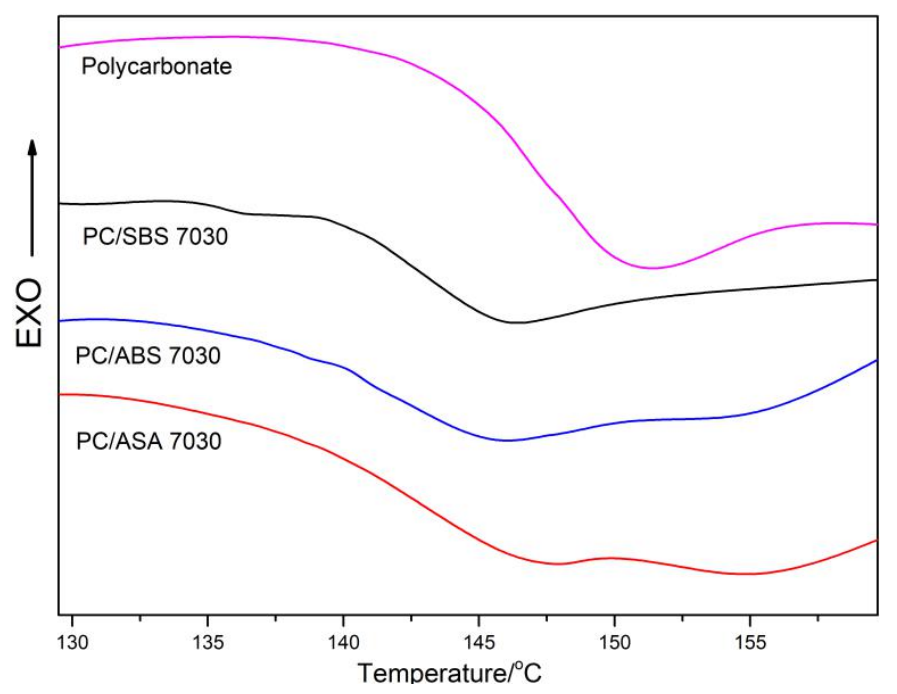

Fig.2 DSC curves of samples

Fig. 3 was the TGA analysis of samples. Compared to the pure polycarbonate, the thermal stabilities of PC blends decreased, the Vicat test (Table 3 ) also indicate that the PC blends became less thermal resistant. This was due to the damage of homogeneities. In addition, the PC/ABS and $\mathrm{PC} / \mathrm{SBS}$ were less thermal stable than PC/ASA, that was because the PC/ABS and PC/SBS contained the butadiene, whose diene segments sensitive to thermal oxidation, under high temperature, the butadiene components were vulnerable and decomposed.

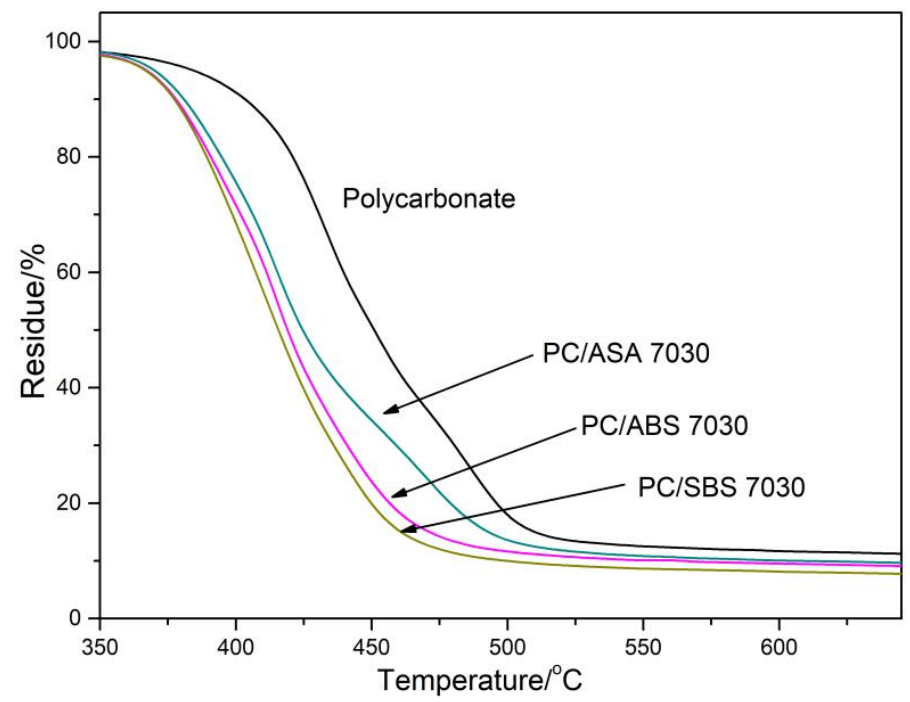

Fig.3 TGA curves of samples

Table 3. Vicat softening temperatures

\begin{tabular}{|c|c|}
\hline & Vicat softening point $/{ }^{\circ} \mathrm{C}$ \\
\hline Polycarbonate & 152 \\
\hline PC/ABS 7030 & 140 \\
\hline PC/ASA 7030 & 146 \\
\hline PC/SBS 7030 & 142 \\
\hline
\end{tabular}




\section{Conclusion}

In this study, three types of polycarbonate/styrene thermoplastics blends (PC/ABS, PC/SBS and PC/ASA) were prepared and characterized. The results showed the mechanical properties of blends were significantly determined by the compositions. To obtain the optimal products, the mass ratios need to be strictly designed.

\section{Reference}

1. J.Alsadi. Systematic review: Impact of processing parameters on dispersion of polycarbonate Composites, and pigment characterized by different techniques. Materials Today: Proceedings. 2020.27(4): 3254-3264.

2. Chunliang Li, Theo Veldhuis, Bart Reuvers, Rafaël J Sablong, Cor E Koning. Fully renewable limonene-derived polycarbonate as a high-performance alkyd resin. Polymer International. 2020.69(1): 24-30.

3. Takayuki Hirai, Kenichi Yagi, Kazuo Okamoto, Yusaku Onochi, and Jumpei Kawada. In Situ Reactive Compatibilization of Polyamide 6 and Polycarbonate Blend by the Catalytic Effect of Phenol Novolac. Ind. Eng. Chem. Res. 2020.59(5): 1855-1861.

4. Riho Nishikawa, Kakeharu Tamaki, Osamu Notoya, Masayuki Yamaguchi. Carbon nanotube localization at interface in cocontinuous blends of polyethylene and polycarbonate. J. Appl. Polym. Sci. 2019.137(19): 48676.

5. Wenqin Lai, Guozhang Wu. Reactive blending and transesterification-induced degradation of isosorbide-based polycarbonate blends. Polymer Degradation and Stability. 2019.162: 201-212.

6. Ikseong Jeon, Seung Woo Lee, Jae Young Jho. Compatibilizing Effect of Poly(methyl methacrylate-co-maleic anhydride) on the Morphology and Mechanical Properties of Polyketone/Polycarbonate Blends. Macromolecular Research. 2019.27: 821-826.

7. Mohammed Arif Poothanari, Priti Xavier, Suryasarathi Bose, Nandakumar Kalarikkal, Cibi Komalan, Sabu Thomas. Compatibilising action of multiwalled carbon nanotubes in polycarbonate/polypropylene (PC/PP) blends: phase morphology, viscoelastic phase separation, rheology and percolation. Journal of Polymer Research. 2019.26: 178.

8. Andrea Mura, FedericaAdamo, Haozhe Wang, Wei Sun Leong, Xiang Ji, Jing Kong. Investigation about tribological behavior of $A B S$ and PC-ABS polymers coated with graphene. Tribology International. 2019.134: 335-340.

9. Fatma Hentati, Ismail Hadriche, Neila Masmoudi, Chedly Bradai. Optimization of the injection molding process for the PC/ABS parts by integrating Taguchi approach and CAE simulation. The International Journal of Advanced Manufacturing Technology. 2019.104: 4353-4363.

10. T.Pandim, T.Doca, A.R.Figueiredo, F.M. AndradePires. Torsional fretting wear experimental analysis of a R3 offshore steel against a PC/ABS blend. Tribology International. 2020.143: 106090.

11. Ruogu Tang, Wenfa Dong, Dingfang Chen. Effects of ABS-g-MAH on morphologies, compatibilities and properties of polycarbonate ternary blends. ChemRxiv. Preprint. 2020. https://doi.org/10.26434/chemrxiv.10058444.v10

12. Wenfa Dong, Ruogu Tang. Preparation and Characterization of Modified Natural Rubber Blends

For Water Purification Treatment Usage. ChemRxiv. Preprint. 2020.

https://doi.org/10.26434/chemrxiv.9971285.v13 